\title{
Highly Porous Platinum Electrodes for Dry Ear-EEG Measurements
}

\author{
M. Eickenscheidt ${ }^{1 *}$, P. Schäfer ${ }^{2}$, Y. Baslan ${ }^{1}$, C. Schwarz ${ }^{3}$, T. Stieglitz ${ }^{14,5}$ \\ 1 Laboratory for Biomedical Microtechnology, IMTEK, University of Freiburg, Freiburg, Germany; \\ 2 Systems Neuroscience \& Neurotechnology Unit, Faculty of Medicine, Saarland University, Homburg, \\ Germany; \\ 3 Hahn-Schickard, Freiburg, Germany \\ 4 BrainLinks-BrainTools, University of Freiburg, Freiburg, Germany \\ 5 Bernstein Center Freiburg, University of Freiburg, Freiburg, Germany \\ * Correspondence: eickenscheidt@imtek.de; Tel.: +49-761-20367636
}

\begin{abstract}
The interest in dry EEG electrodes has increased in recent years and especially as everyday suitability earplugs for measuring drowsiness or focus of auditory attention. However, the challenge is still the need for a good electrode material, which is reliable and can be easily processed for highly personalized applications. Laser processing as used here is a fast and very precise method to produce personalized electrode configurations that meet the high requirements of in-ear EEG electrodes, for example. The arrangement of the electrodes on the very flexible and compressible mats allows an exact alignment of the electrodes to the ear mold and contributes to a high wearing comfort, as no edges or metal protrusions are present. For better transmission properties, an adapted coating process for surface enlargement of platinum electrodes is used, which allows easy control of the thickness and growth form of the porous layer. The porous platinum-copper alloy is chemically very stable, shows no exposed copper residues and enlarges the effective surface area by 40. In a proof-of-principle experiment, these porous platinum electrodes could be used to measure the Berger effect in a dry state using just one ear of a test person. Their signal-to-noise ration and frequency transfer function is comparable to gel-based silver/silver chloride electrodes.
\end{abstract}

Keywords: Ear-EEG; Laser Structuring; Porous Platinum; Berger effect

\section{Introduction}

Measurement of electrography of the brain waves or muscle actions is a simple and excellent way to gain insight into a person's condition or behavior. In particular, non-invasive methods such as electroencephalography (EEG) can provide an overview of neurobiological and cognitive states. This measurement method is relatively compact, but requires a large number of electrodes to be attached to the scalp and is usually performed in a clinical environment, as the preparation and positioning of the electrodes have a decisive influence on the usability and quality of the data. Longterm measurements over days or real-life measurements carried out by the test person are thus excluded [1]. Such a constant contact to the person would offer possibilities such as monitoring patients during sleep [2], detecting driver drowsiness [3] or attention focusing of auditory attention [4] for the improvement of hearing aids.

In order to overcome this limitation, ear-EEG measurements have been studied in recent years, which are characterized by simple handling and unobtrusiveness [5-7]. In this approach, several electrodes are realized on an earplug and conduct the electrical cortical signal in the auditory canal and the auricle. Concepts range from flexible silicone plugs with conductive silicones or coatings [810] to hard shells that are adapted to the respective ears with individually attached electrodes that have been cut out or metal pastes that have been applied manually $[2,12]$. Especially, porous silver chloride is often used, which degrades over time, and conductive gel [11] must be used for acceptable measurements, which excludes long-term measurements, as this dries out over time. These gel-based silver/silver chloride electrodes are state-of-the-art and better handling of dry electrodes does not 
outweigh inferior signal-to-noise levels. To enable dry electrode measurements without additional gels, the active electrode surfaces are enlarged by i.e. iridium oxide and Poly(3,4ethylenedioxythiophene)-PEDOT depositions or needle like structures [10,13-16]. The long-term stability and the manual character of the production as well as the undefined electrode sizes and surfaces are thus the limiting factors for the accurate and reliable production and design of the ear electrodes.

In this study, different materials are compared due to their electrochemical properties and a platinum $(\mathrm{Pt})$ based ear-electrode is presented with highly increased active surface with minimal impedance in the relevant frequency domain. An electrodeposition process is used which creates highly porous platinum surfaces through the iterative co-deposition of $\mathrm{Pt}$ and copper $(\mathrm{Cu})$, whereby $\mathrm{Cu}$ is released again after each cycle [17]. Thus, defined surface structures of different heights can be produced. The manufacturing process must allow a quick adaptation to the physiology of the test persons with a high degree of precision and reliability. For this purpose, prototype processes based on laser cutting were used, structuring individual electrode configurations for personalized earplugs. For the first tests, electrode mats with electrodes of different materials and sizes were produced. The distance between the electrodes was adapted to the personalized imprints of the ear canals and placed on earplugs. The Berger effect was chosen for validating the usability of the porous electrode material.

\section{Materials and Methods}

\subsection{Prototype fabrication}

The electrode array for the otoplastics (Figure 1A) was prepared using a fast laser-aided manufacturing process to gain highest design flexibility. The method used multi-layer processing to achieve the personalized design and was based on previously published processes [18,19]. It entailed the spin coating of a $70 \mu \mathrm{m}$ thick MED1000 silicone layer (NuSil, Carpinteria, USA) on a flat carrier substrate. Subsequently, different metal sheets (Platinum, Silver, MP35N all of $25 \mu \mathrm{m}$ thickness) were laminated on the pre-cured silicone layer and the electrode design was cut out with a picosecond laser (Coherent Inc., Santa Clara, CA), using the parameters suited for the material type and thickness. The excess metal was peeled off and the silicone layer fully cured, forming a strong bond with the metal structures. The conductor paths were designed in a meandering shape in order to maintain the high flexibility of the system (Figure 1B). A topside coating was performed with a chemical vapor deposition system (Dimer DPX-C, Coater PDS 2010, Speciality Coating Systems Inc., Indianapolis, IN, USA) with $10 \mu \mathrm{m}$ Parylene-C (Figure 1C). The prototyping laser process was used again to pattern the Parylene-C layer to expose the active sites of the electrodes on one end, and the connector soldering pads on the other, while leaving the conducting paths between them isolated. Finally, the outlines were cut with the same laser, to release the probe.

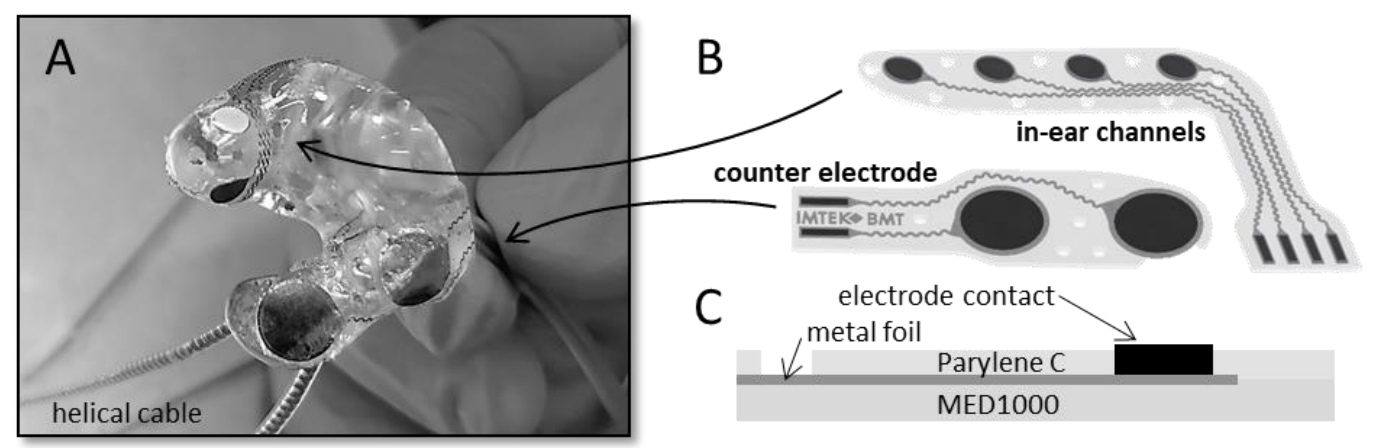

Figure 1. (A) Image of the fully assembled ear-EEG electrode with four electrodes in the ear canal ( $3 \mathrm{~mm}$ diameter) and two large counter electrodes with $6 \mathrm{~mm}$ diameter. The in-ear electrode arrays are personalized laser design files with adjusted electrode spacing for an equidistant electrode spacing in the auditory channel (B). The two electrode mats have the same layering (C); a medical grade silicone laminated with a structured metal foil, encapsulated with $10 \mu \mathrm{m}$ Parylene $\mathrm{C}$. 


\subsection{Soldering, cleaning and characterization of electrodes}

After fabrication, the electrode arrays were assembled with a connector by soldering enameled copper or MP35N helical winded cables to the soldering pads, and the single wires to a pin header for addressing each electrode separately. The electrode array was cleaned thoroughly with ethanol and de-ionized water, to remove residues or dust particles from the surface. In addition to washing, the electrodes were also cleaned electrochemically by running 15 cycles of cyclic voltammetry at a rate of $250 \mathrm{mVs}-1$ within a range of $-600 \mathrm{mV}$ to $+900 \mathrm{mV}$ in a phosphate-buffered saline solution. All electrochemical procedures were performed with a three-electrode setup using a potentiostat and a frequency analyzer (Solartron 1260\&1287, Solartron Analytical, Farnborough, United Kingdom). The assembled electrodes were characterized before and after a subsequent electrodeposition, in order to estimate their effective surface area as well as to analyze their frequency response. To estimate the area, cyclic voltammetry was performed for 3 cycles at a rate of $100 \mathrm{mVs}^{-1}$ within a window of $700 \mathrm{mV}$ to $+1000 \mathrm{mV}$ vs. SCE. The evaluation of the charge delivery capacity (CDC) was performed with Origin (OriginPro 2019, Northhampton, MA, USA) by calculating the enclosed polygon area of the last cycle. As for the frequency response, the impedance in a range of $0.1 \mathrm{~Hz}$ to $100 \mathrm{kHz}$ was determined, at a rate of 5 steps/decade and a $20 \mathrm{mV}$ AC amplitude. Scanning electron micrographs of the electrode morphology and focused ion beam cuts were made with a Scios 2 (Thermo Fischer Inc., MA, USA). The chemical analysis of the surfaces was performed by a X-Ray Fluorescence Spectrometer ( $\mu$-XRF M4 Tornado, Bruker Corporation, MA, USA) in vacuum. The beam had a strength of $50 \mathrm{kV}$ at $600 \mu \mathrm{A}$ and a spot size of $20 \mu \mathrm{m}$. After $30 \mathrm{~s}$ measurement, the atomic concentrations were analyzed with the internal software.

The finalized electrodes were glued onto personalized otoplastics. Ear impressions of the respective test person were used to produce hard shells from acrylic resin (Sivantos $\mathrm{GmbH}$, Erlangen, Germany). The shells were cleaned with isopropanol and the silicone side of the electrode mat was bonded to the shell with ethyl-2-cyanoacrylate adhesive (RS Components SAS, France). Two large electrodes were positioned in the concha and four smaller ones evenly distributed around the ear channel section (the electrode design were adapted to the otoplastic). The electrodes were placed on the outer edge of the earpiece in order to allow an electrode measurement as deep as possible in the ear channel. The connections of the mats and the cables were fixed on the outside of the shell with MED1000.

\subsection{Electrodeposition and electroplating}

The electrodeposition process used in this work was adapted from Frei et al. [17], with minor changes to suit the application. The experiments were conducted in a three-electrode setup using the Solartron Analytical 1287A potentiostat function. The reference electrode was a saturated calomel electrode (SCE, KE 11, Sensortechnik Meinsberg, Ziegra-Knobelsdorf, Germany) and for the counter electrode a platinum mesh $(0.06 \mathrm{~mm}$ wire diameter $/ 99.9 \%$, Chempur, Karlsruhe, Germany) connected to a platinum wire (0.1 mm diameter, Chempur, Karlsruhe, Germany) was used. The electrolyte consists of $0.5 \mathrm{M}$ sulfuric acid (TitriPUR, Merck, Darmstadt, Germany) containing $0.02 \mathrm{M} \mathrm{Cu}_{2} \mathrm{SO}_{4}$ (Merck, Darmstadt, Germany) and $0.02 \mathrm{M} \mathrm{H}_{2} \mathrm{PtCl}_{6}$ (Chempur, Karlsruhe, Germany). During the experiment, the electrolyte was kept in a nitrogen atmosphere, which was established by first bubbling nitrogen through the solution for 20 minutes and then purging it continuously on the surface of the electrolyte. Once the solution was saturated with the nitrogen, the electrodeposition process was executed by simultaneously depositing $\mathrm{Cu}$ and $\mathrm{Pt}$ from the electrolyte, by applying a deposition potential of $-500 \mathrm{mV}$ vs. SCE for $9 \mathrm{~s}$ to the working electrode. The deposition was followed by a selective dissolution of copper at a potential of $+700 \mathrm{mV}$ vs. SCE for $4.5 \mathrm{~s}$. This alternating process was repeated 50 times. To counteract possible accumulation of gas bubbles on the active surface, the medium was constantly moved with a magnetic stir bars.

Silver electrodes were chlorinated in a potassium chloride solution $(3 \mathrm{~mol})$ with a single $\mathrm{Ag} / \mathrm{AgCl}$ counter electrode, by passing a DC-current through the electrode at a rate of $1 \mathrm{~mA} / \mathrm{cm}^{2}$ of surface area for 15 seconds. 

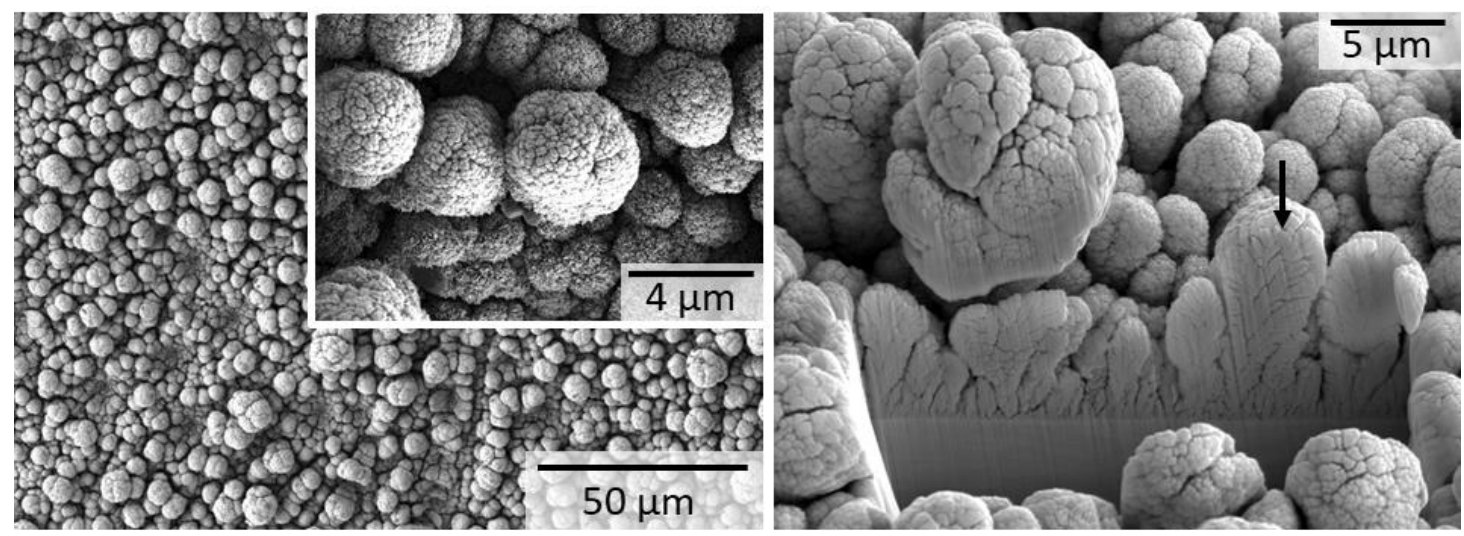

Figure 2. Scanning electron microscopy of the full-surface metal coating with porous platinum after alloying with copper and alternating dealloying. The surface shows different forms of growth (left). In deeper layers, finger-like structures can be seen, which become more cauliflower-like towards the top (insert). A cross-section with the focused ion beam shows channel structures up to the platinum foil (left). The height of the average structures (arrow) is around $10 \mu \mathrm{m}$.

\subsection{In-Ear Electroencephalography Measurement}

Before each experiment, the auricle and the anterior part of the external auditory canal of the test person were cleaned with a cotton swab and alcohol. This area was then treated with a peeling gel using a cotton swab to remove old and dead skin residues. Finally, the exfoliating gel was thoroughly removed and the area was cleaned again with alcohol. In the case of $\mathrm{Ag} / \mathrm{AgCl}$ probes, gel was applied to the electrode surfaces in a dot-shaped manner. The prepared electrode was then inserted into the prepared ear and the electrodes connected to the EEG amplifier (g.USBamp, g.tec medical engineering $\mathrm{GmbH}$, Austria). During the measurement, the subject sat quietly on a chair in a quiet and non-illuminated room. In a first measurement, the test person opened his eyes for more than 30 seconds and fixes a cross on the wall in front of him. In a second measurement, the respondent closed his eyes for 30 seconds. At the same time, the EEG was recorded at a sampling rate of $512 \mathrm{~Hz}$. The spectral density was analyzed by the multitaper method [20]. The time signals of all single channels were filtered with a set of Slepian sequences of a window half-bandwidth of 4 and the final spectrum was obtained by averaging all convoluted data. The band powers were calculated by integrating a parabolic approximation between the sample points of a chosen frequency spectrum (Simpson's rule). All calculations were performed with customized programs in Python.

\section{Results}

\subsection{Electrode preperation}

A laser-assisted ablation process realizes the patient-specific manufacture of the electrode arrangement. For an equidistant arrangement of the four electrodes in the ear canal, the radius of the personalized otoplastic was first measured and the electrodes structured accordingly on the silicone mat. The measuring electrodes with a diameter of $3 \mathrm{~mm}$ were intended to be placed in the external auditory channel and two further counter electrodes with a diameter of $6 \mathrm{~mm}$ in the concha at the level of the cymba. The conductive paths were guided in a meandering pattern to minimize stress on the conductive paths when they were attached to the hard shell. The solder joints between the electrode mat and the helically wound cable were fixed and insulated with epoxy adhesive (Figure 1). The highly flexible cable relieved the tension on the fixation and was not as susceptible to movements as straight cables. 
Before the polymer-based electrodes were glued onto the earplug, the surfaces were treated and, in particular, the platinum-copper alloy was deposited. This results in different microstructures that were created depending on the number of cycles of iterative alloying and delegation of platinum and copper. A deposition with 50 iterations has proven to be advantageous to obtain a 5 to $20 \mu \mathrm{m}$ thick layer of platinum. The structures change from initially bicontinuous ligament-channel structure to larger cauliflower like structures (Figure 2).

Table 1. XRF Measurement of three pristine platinum electrodes with electrodeposited platinumcopper alloy. Two of these electrodes where treated with different etching methods for copper (electrochemical dealloying and wet chemical etching).

\begin{tabular}{ccc}
\hline Electrode treatment & $\mathbf{P t}[\mathbf{w t} \%]$ & $\mathbf{C u ~ [ w t \% ]}$ \\
\hline pristine & $87.16 \pm 0.09$ & $12.84 \pm 0.09$ \\
Dealloying $30 \mathrm{~min}$ & 88.60 & 11.40 \\
$\mathrm{Na}_{2} \mathrm{~S}_{2} \mathrm{O}_{8} 20 \mathrm{~min}$ & 87.83 & 12.17 \\
\hline
\end{tabular}

XRF studies show a high copper content of $12 \mathrm{wt} \%$ in the applied layer despite alternating dealloying (see Table 1). This layer cannot be spatially assigned to any structure and was also not optically recognizable. Two attempts to reduce the copper content were made without any effect. On the one hand the negative potential of $-600 \mathrm{mV}$ vs. SHE was kept for 30 minutes after deposition, on the other hand the electrodes were etched in a standard etching bath for printed circuit boards with heated $\left(80^{\circ} \mathrm{C}\right)$ sodium persulfate $\left(\mathrm{Na}_{2} \mathrm{~S}_{2} \mathrm{O}_{8}\right)$ for 20 minutes. Subsequent XRF measurements show only small variations that were not significant.

\subsection{Electrochemical electrode characterisation}

All electrodes were built in the layered structure of PDMS - metal foil - Parylene C (Figure 1C) in different designs, whereby the accuracy of the ablative laser process gave the real spot sizes. The properties of the electrodes were determined in PBS solution to simulate a physiological measurement condition (Figure 3). Smooth platinum electrodes show a typical impedance behavior of a Randles cell with the access resistance at high frequencies and a characteristic increase in impedance to low frequencies given by the active surface and electrode matter (Figure 3A).
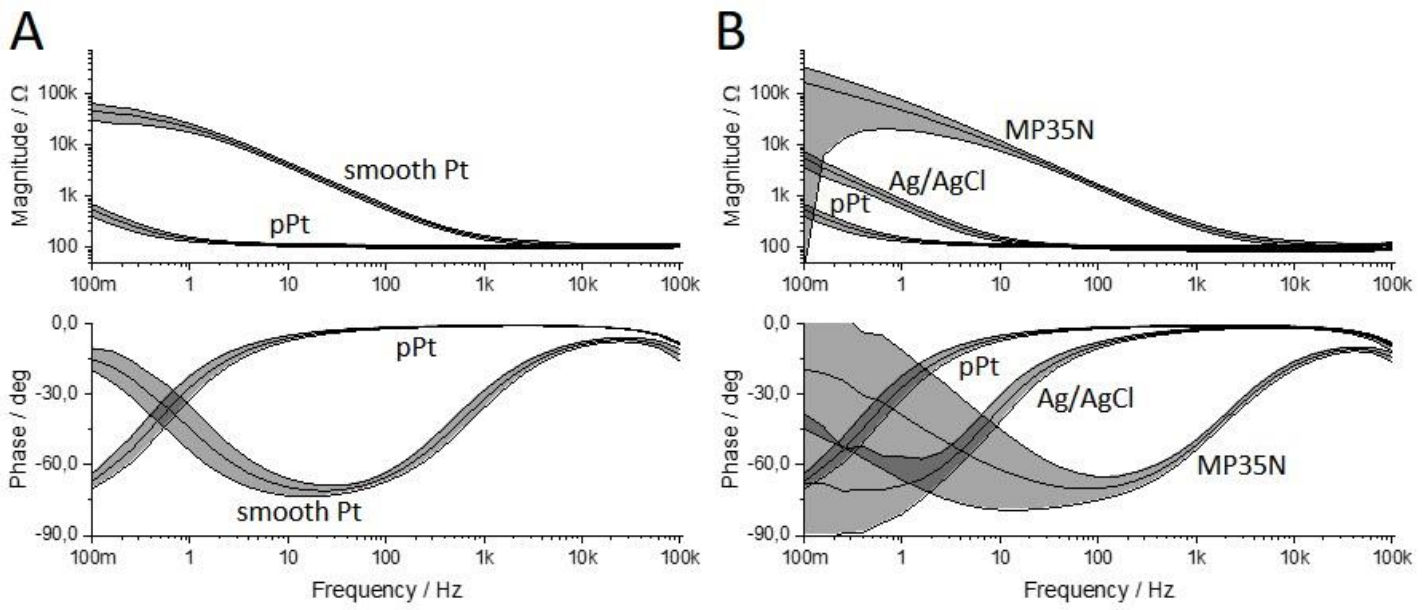

Figure 3. Electrochemical impedance measurement of different electrode materials. (A) Smooth platinum electrodes before and after electrochemical deposition of porous platinum $(\mathrm{N}=10)$. (B) comparison of $\mathrm{MP} 35 \mathrm{~N}(\mathrm{~N}=7), \mathrm{Ag} / \mathrm{AgCl}(\mathrm{N}=7)$ and porous platinum (pPt) electrodes of the same geometric area (3mm diameter). 
The access resistance is given by the geometric expansion of the electrode and therefore it is independent of the electrode material or the roughening (Figure 3A/B). The deposition of platinum copper or the chlorination of the electrodes was performed using the Solartron setup. The porous surfaces show a significant reduction of the impedance at low frequencies, which leads to a purely resistive behavior at frequencies higher than $1 \mathrm{~Hz}$ (Figure 3A, phase). A similar behavior can be observed in the chlorination of silver foils, whereas the reduction of impedance was not as strong as in the surface enlargement by platinum. The reference material MP35N, which is the gold-standard material due to its biocompatibility and stability, has an even earlier increase in complex impedance than smooth platinum foils.
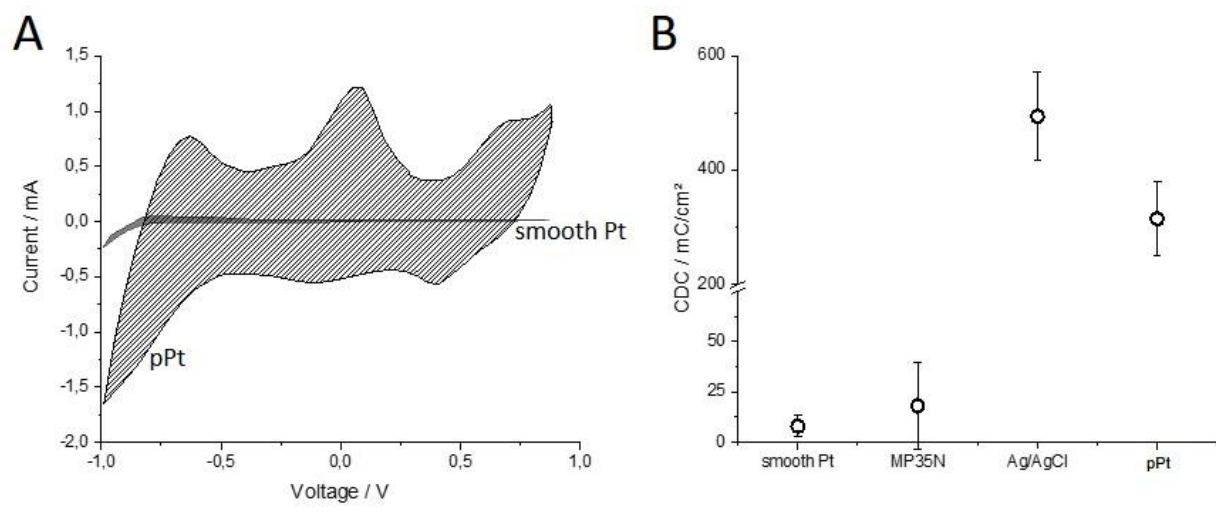

Figure 4. Cyclic voltammetry measurement. A) Smooth platinum electrodes before and after electrochemical deposition of porous platinum (mean of 10 electrodes each). B) Comparison of the charge delivery capacity (CDC) of MP35N (N=7), $\mathrm{Ag} / \mathrm{AgCl}(\mathrm{N}=7)$, smooth and porous platinum (pPt) electrodes $(\mathrm{N}=10$ each) of the same geometric area.

Another electrochemical characterization of the electrodes is cyclic voltammetry, which indicates the current flow at a linearly varying voltage between two reversal points (Figure 4). Here the dissipative and reactive currents were superimposed across the electrode interface. Both components depend on the effective surface area of the electrodes and increase with the roughness of the surface (Figure 4A). A quantitative evaluation of electrochemical electrode surface properties is the charge delivery capacity (CDC), which is calculated by the enclosed area of the curve of a cycle and is divided by the slope of the applied ramp to obtain a value for the charge transfer in the applied voltage window. These values were normalized to the geometric electrode size. A comparison of CDCs shows large differences between the materials used and surface conditions (Figure 4B). For example, the rolled and smooth surfaces of platinum and MP35N show a CDC of 8 and $18 \mathrm{mC} / \mathrm{cm}^{2}$ respectively. A surface enlargement of the porous platinum electrodes results in a 40 -fold enlargement of the CDC to $315 \mathrm{mC} / \mathrm{cm}^{2}$. Silver/silver chloride electrodes also showed a strongly enlarged CDC $\left(495 \mathrm{mC} / \mathrm{cm}^{2}\right)$ too, which was due to the change in surface structure, but mainly to the reactive property of the metal salt in contact with the electrolyte.

\subsection{EEG Measurment}

A promising application for the greatly enlarged electrode surfaces is the recording of biological signals in contact with the skin. As proof of principle, the EEG signals were recorded at the auricle and in the ear canal. Two pairs of ear EEG electrodes were made for one person, one with platinumcopper alloy and one made of chlorinated silver foil. The ear molds were easy to use and can be inserted by the individual independently. Towards a real world scenario of the platinum electrodes, external reference and counter-electrodes, e.g. on the lobulus, $\mathrm{Cz}$, or low mastoids have been omitted. The data refer exclusively to measurements from 4 electrodes within the auditory canal referenced to the electrodes in the concha on the same side of the head. To check the functionality, the Berger effect was measured in a shielded EEG laboratory without external disturbances, where the test person 
kept the eyes open and closed for 30 seconds each. A successful differentiation of the frequency spectra in the alpha band [8-12 Hz] was possible with both materials, whereas conductive gel had to be used with silver/silver-chloride electrodes while measurements with the porous platinum electrodes were conducted in dry condition. Furthermore, for the measurements with the silver electrodes, the low mastoid on the same side of the head was used as a reference. The mean values and standard deviations of the frequency spectra across all 4 electrodes in the auditory canal show an increase in power in the alpha band when the eyes were closed (Figure 5). Thus, on average, an increase in power of $2.4 \pm 1.8 \mu \mathrm{V}^{2}$ can be expected for chlorinated silver electrodes and $4.5 \pm 3.7 \mu \mathrm{V}^{2}$ for porous platinum electrodes. The high variances reflect the different electrode performances. For example, a selected electrode with a ratio of up to 4.4 can be found in the chloride silver device, whereas the electrodes in the platinum system had a median change of 2.43 power in the alpha band (Table 2). In general, however, the power density of silver electrodes was one order of magnitude higher than that of porous platinum electrodes. Also, a change of the increased power in the beta band with open eyes was only visible with the silver electrodes. On the other hand, the signal variance of the platinum electrodes was significantly lower.
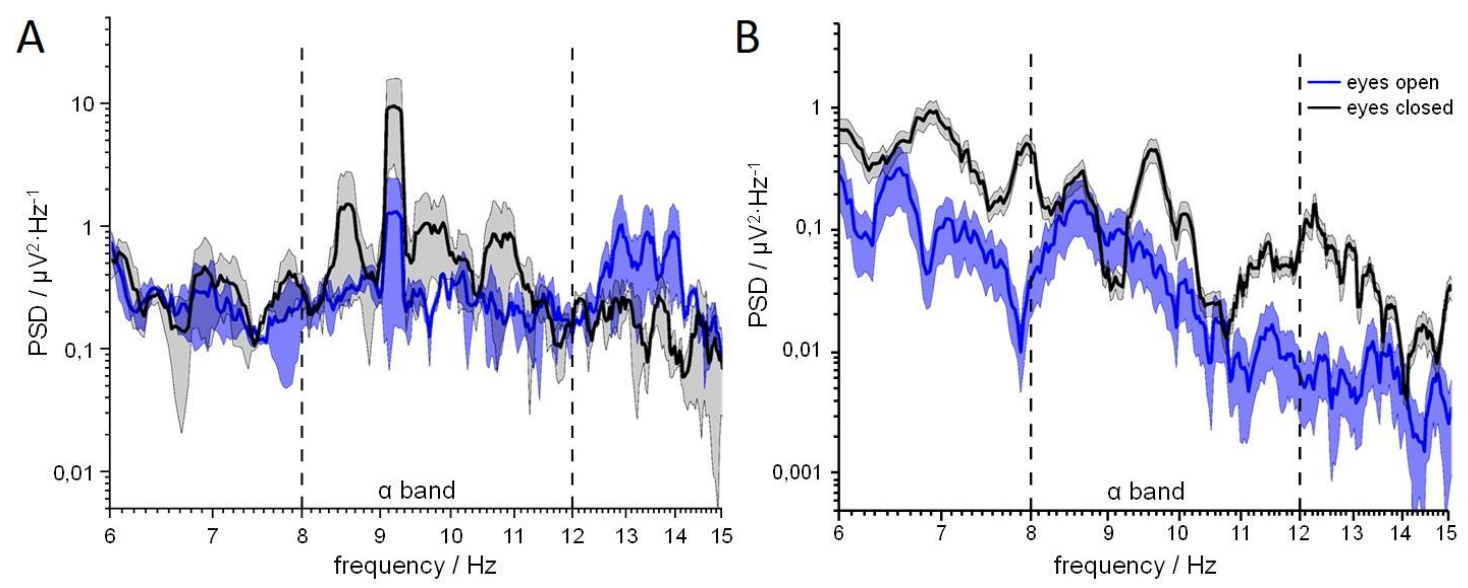

Figure 5. Averaged frequency spectra of 4 electrodes in the auditory canal and their standard deviation (transparent intervall). The power density with closed eyes (black) is higher in the alpha band between 8 and $12 \mathrm{~Hz}$ than with open eyes (blue curve). This Berger effect can be observed with chlorinated silver electrodes using conductive gel (A) and dry porous platinum electrodes (B).

Table 2. The alpha power is calculated in the bandwidth between 8 and $12 \mathrm{~Hz}$ for 30 seconds closed eyes. The Berger effect is the ratio between the alpha powers of closed and opened eyes.

\begin{tabular}{ccc}
\hline porous $\mathbf{P t}$ & $\alpha$ power $\left[\mu \mathrm{V}^{2}\right]$ & Berger effect \\
\hline channel 1 & 0,603 & 2,43 \\
channel 2 & 0,643 & 2,28 \\
channel 3 & 0,606 & 2,44 \\
channel 4 & 0,362 & 10,9 \\
\hline
\end{tabular}

\section{Discussion}

The two-step process presented here for manufacturing earplugs with passive electrodes for EEG measurements was designed for rapid personalization. Depending on the hard shell of the test person, the electrode spacing and positioning can be freely selected and can be produced in the prototype laser process with little effort. The MEMS process thus offers more design freedom and better control than manual printing processes and cabling $[5,8]$. The small thickness of the entire electrode mat allows the otoplastic to be worn comfortably because it does not protrude as far as printed electrodes do. The $10 \mu \mathrm{m}$ thick cover layer also allows good contact with the skin - especially in the case of rough electrodeposited platinum - whereas the insulation between the individual 
electrodes was maintained. The large thicknesses of the layers are a great advantage compared to alternative coatings such as iridium oxide (IrOx) [21], as a flat surface of electrode material and insulator can be achieved. In a second step, the various post-treatments of the electrodes, the connections and the positioning on the earplug are independent of production, which also allows a higher degree of freedom from the choice of material to the overall concept.

The surface enlargement by the iterative electroplating of platinum and copper and the subsequent dealloying by removal of copper atoms leads to a strong enlargement of the active surface of the electrodes. The area of the cyclic voltammetry measurement shows a magnification by a mean factor of 39.41. In previous studies a higher value of up to a factor of 1000 was shown, whereby these values were determined by hydrogen or carbon dioxide stripping [17]. The measurement presented here was intentionally performed with PBS and a higher scan rate of $100 \mathrm{mV} / \mathrm{sec}$ as this provides a better insight into the effective surface in the application scenario. In the intended EEG measurement system, dissolved salts in the form of sweat also form the decisive phase boundary. Due to their larger shape and hydrate shell, however, salts have a much larger dimension than hydrogen ions and therefore only a fraction of the porous surface contributes to the interface. The Faraday resistance of the electrode - which is e.g. a chemical reaction of platinum with hydrogen or oxygen - was negligible in the relevant frequency range (compare impedance measurements). The capacitance of the electrode has been increased so that it short-circuits the phase boundary and only the access resistance of the electrolytes was of importance. The access resistance depends on the geometric shape and the composition of the electrolyte, namely the salt concentrations.

All plated electrodes (diameter $3 \mathrm{~mm}$ ) used here were below $5 \mathrm{kOhm}$ at $20 \mathrm{~Hz}$ and therefore compatible with standard medical EEG systems. In this study platinum was compared to MP35N nickel-cobalt base alloy, which is widely used in biomedical applications, which has an even higher impedance in the rolled state than platinum. Secondly, silver/silver-chloride electrodes, which are used as conventional EEG electrodes, show comparable electrical characteristics to the porous platinum. However, silver and gold electrodes should only be used about 10-20 times [22]. The silver electrodes were not homogeneously electroplated and therefore only a slight improvement of the EIS can be seen. Even if the charge capacity were the highest, what would be expect for a non-polarizable electrode. The increase in impedance at low frequency was therefore partly due to the material [22] and partly to the inhomogeneous coating. Furthermore, silver/silver chloride was heavily affected by exposing it to sunlight. Which requires special storage of the electrodes. A promising surface enlargement of the electrodes with iridium oxide for an application as dry electrode was also used, whereby only a surface enlargement by a factor of 15 is to be expected here [21]. These nanoporous very thin layers (as well as platinum grass [21] or carbon nano tubes [6,8,9]) show excellent properties as implantable electrodes, especially having small diameters. However, they may not have the necessary stability and thickness to provide a good and long lasting contact as a re-usable patch electrode, which is to be expected with the microstructure of the porous platinum proposed here.

The XRF measurements show a significant copper content in the bulk of the electroplated material, which remains in the metallic compound. Excessive and selective etching of copper (chemical and electrochemical) shows no change, so it is suspected that the copper atoms were not exposed on the surface of the porous material but were firmly enclosed by metal lattices. For longterm applications of the material in contact with the skin, a low concentration of copper is nevertheless to be assumed, which could get into the body percutaneously [23] and lead to irritation or allergies. However, pure copper material is commonly used as intrauterine device to regulate fertility or as dental implants, and if the concentrations are very low -as in the current study- the risk to the patient is minimal. With an overestimation volume of the grown platinum copper alloy of $0.28 \mathrm{~mm}^{3}(10 \mu \mathrm{m}$ thick structure $)$ a recording spot has a maximum of $0.78 \mathrm{mg}$ copper. For comparison, the Food and Nutrition Board gives a NOAEL value of $10 \mathrm{mg} / \mathrm{d}$ for an adult. In a possible application, however, further tests must be carried out.

A further possible application for the flexible thin electrode mats are patch electrodes for the skin. Measuring the in-ear EEG was chosen, as this is a highly interesting location, which on the one hand is easily accessible hub of information and on the other hand makes high demands on the 
personalization of the device [24]. The measurements of the Berger effect were successful, although there were some differences to the known signals [25], which are normally picked up over the entire head area ( $\mathrm{C} 3$ or $\mathrm{C} 4$ and adjacent electrodes). In addition, the choice of the reference electrode on the same ear in the auricle is a challenging choice [26]. The derivation of the EEG signal referenced to e.g. the mastoid gives better results like in the case of the chlorinated silver electrodes. However, the silver electrodes showed unsatisfactory measurement results in dry condition. Further improvements can be expected if the electrodes of both ears are used and the signals are referenced to the other side of the head [26].

\section{Conclusions}

Towards a reliable and resistant electrode material for skin patch electrodes with high signal-to noise-ratio, the platinum-copper alloy presented here is a promising candidate. The simple and controllable production of the coatings by electrochemical processes on personalized structured metal foils is a decisive advantage over costly cleanroom processes and deposition of mostly thin functional layers. The porous platinum layers show appropriated measuring properties as dry EEG electrodes. Especially in the relevant frequency band $0.1 \mathrm{~Hz}$ up to $100 \mathrm{~Hz}$ but also beyond. Alternative materials such as smooth platinum, MP35N or chlorinated silver foils have higher impedances and coupling properties. The chemical inertness and the low degradation of the material compared to e.g. chlorinated silver electrodes suggests for a long-lasting and reliable use of the material.

\section{Supplementary Materials: N.A.}

Author Contributions: Conceptualization ideas M.E., D.S. and T.S.; investigation Y.B., P.S. and C.S.; formal analysis M.E., P.S., Y.B. and C.S.; writing-original draft preparation M.E., P.S. and Y.B.; writing-review and editing D.S. and T.S.

Funding: This research received no external funding

Acknowledgments: The author would like to thank Dr. Yi Thomann for her assistance with SEM and FIB pictures, and Maxi Frei and Sven Kerzenmacher for their help to setup the deposition process. The authors thank Sivantos $\mathrm{GmbH}$ for manufacturing and delivery of the otoplastics

Conflicts of Interest: The authors declare no conflict of interest.

\section{References}

1. Mihajlovic, V.; Grundlehner, B.; Vullers, R.; Penders, J. Wearable, wireless EEG solutions in daily life applications: What are we missing? IEEE J. Biomed. Health Inform. 2015, 19, 6-21, doi:10.1109/JBHI.2014.2328317.

2. Mikkelsen, K.B.; Tabar, Y.R.; Kappel, S.L.; Christensen, C.B.; Toft, H.O.; Hemmsen, M.C.; Rank, M.L.; Otto, M.; Kidmose, P. Accurate whole-night sleep monitoring with dry-contact ear-EEG. Sci. Rep. 2019, 9, 16824, doi:10.1038/s41598-019-53115-3.

3. Bernarding, C.; Herbig, M.-C.; Pratapa, N.A.; Choquet, V.; Angermayer, J.; Strauss, D.J. Time-Resolved In-Vehicle Drowsiness Monitoring Using Multimodal Electrophysiological Data. In 2019 9th International IEEE/EMBS Conference on Neural Engineering (NER). 2019 9th International IEEE/EMBS Conference on Neural Engineering (NER), San Francisco, CA, USA, 20.03.2019 - 23.03.2019; IEEE, 2019; pp 457-460, ISBN 978-1-5386-7921-0. 
4. Fiedler, L.; Wöstmann, M.; Graversen, C.; Brandmeyer, A.; Lunner, T.; Obleser, J. Single-channel in-earEEG detects the focus of auditory attention to concurrent tone streams and mixed speech. J. Neural Eng. 2017, 14, 36020, doi:10.1088/1741-2552/aa66dd.

5. Mikkelsen, K.B.; Kappel, S.L.; Mandic, D.P.; Kidmose, P. EEG Recorded from the Ear: Characterizing the Ear-EEG Method. Front. Neurosci. 2015, 9, 438, doi:10.3389/fnins.2015.00438.

6. Hoon Lee, J.; Min Lee, S.; Jin Byeon, H.; Sook Hong, J.; Suk Park, K.; Lee, S.-H. CNT/PDMS-based canaltyped ear electrodes for inconspicuous EEG recording. J. Neural Eng. 2014, 11, 46014, doi:10.1088/17412560/11/4/046014.

7. Bleichner, M.G.; Lundbeck, M.; Selisky, M.; Minow, F.; Jäger, M.; Emkes, R.; Debener, S.; Vos, M. de. Exploring miniaturized EEG electrodes for brain-computer interfaces. An EEG you do not see? Physiol. Rep. 2015, 3, doi:10.14814/phy2.12362.

8. Lee, J.H.; Hwang, J.-Y.; Zhu, J.; Hwang, H.R.; Lee, S.M.; Cheng, H.; Lee, S.-H.; Hwang, S.-W. Flexible Conductive Composite Integrated with Personal Earphone for Wireless, Real-Time Monitoring of Electrophysiological Signs. ACS Appl. Mater. Interfaces 2018, 10, 21184-21190, doi:10.1021/acsami.8b06484.

9. Chlaihawi, A.A.; Narakathu, B.B.; Emamian, S.; Bazuin, B.J.; Atashbar, M.Z. Development of printed and flexible dry ECG electrodes. Sensing and Bio-Sensing Research 2018, 20, 9-15, doi:10.1016/j.sbsr.2018.05.001.

10. Kappel, S.L.; Rank, M.L.; Toft, H.O.; Andersen, M.; Kidmose, P. Dry-Contact Electrode Ear-EEG. IEEE Trans. Biomed. Eng. 2019, 66, 150-158, doi:10.1109/TBME.2018.2835778.

11. Kappel, S.L.; Kidmose, P. Study of impedance spectra for dry and wet EarEEG electrodes. Conf. Proc. IEEE Eng. Med. Biol. Soc. 2015, 2015, 3161-3164, doi:10.1109/EMBC.2015.7319063.

12. Kidmose, P.; Looney, D.; Jochumsen, L.; Mandic, D.P. Ear-EEG from generic earpieces: A feasibility study. Conf. Proc. IEEE Eng. Med. Biol. Soc. 2013, 2013, 543-546, doi:10.1109/EMBC.2013.6609557.

13. Li, G.; Wang, S.; Duan, Y.Y. Towards gel-free electrodes: A systematic study of electrode-skin impedance. Sens. Actuator B Chem. 2017, 241, 1244-1255, doi:10.1016/j.snb.2016.10.005.

14. Lopez-Gordo, M.A.; Sanchez-Morillo, D.; Pelayo Valle, F. Dry EEG electrodes. Sensors (Basel) 2014, 14, 12847-12870, doi:10.3390/s140712847.

15. Fonseca, C.; Silva Cunha, J.P.; Martins, R.E.; Ferreira, V.M.; Marques de Sá, J.P.; Barbosa, M.A.; Martins da Silva, A. A novel dry active electrode for EEG recording. IEEE Trans. Biomed. Eng. 2007, 54, 162-165, doi:10.1109/TBME.2006.884649.

16. Chen, Y.-H.; Beeck, M. op de; Vanderheyden, L.; Carrette, E.; Mihajlović, V.; Vanstreels, K.; Grundlehner, B.; Gadeyne, S.; Boon, P.; van Hoof, C. Soft, comfortable polymer dry electrodes for high quality ECG and EEG recording. Sensors (Basel) 2014, 14, 23758-23780, doi:10.3390/s141223758.

17. Frei, M.; Köhler, C.; Dietel, L.; Martin, J.; Wiedenmann, F.; Zengerle, R.; Kerzenmacher, S. Pulsed Electrodeposition of Highly Porous Pt Alloys for use in Methanol, Formic Acid, and Glucose Fuel Cells. ChemElectroChem 2018, 5, 1013-1023, doi:10.1002/celc.201800035.

18. Vomero, M.; Oliveira, A.; Ashouri, D.; Eickenscheidt, M.; Stieglitz, T. Graphitic Carbon Electrodes on Flexible Substrate for Neural Applications Entirely Fabricated Using Infrared Nanosecond Laser Technology. Sci. Rep. 2018, 8, 14749, doi:10.1038/s41598-018-33083-w.

19. Mueller, M.; La Oliva, N. de; Del Valle, J.; Delgado-Martínez, I.; Navarro, X.; Stieglitz, T. Rapid prototyping of flexible intrafascicular electrode arrays by picosecond laser structuring. J. Neural Eng. 2017, 14, 66016, doi:10.1088/1741-2552/aa7eea. 
20. Gramfort, A.; Luessi, M.; Larson, E.; Engemann, D.A.; Strohmeier, D.; Brodbeck, C.; Goj, R.; Jas, M.; Brooks, T.; Parkkonen, L.; et al. MEG and EEG data analysis with MNE-Python. Front. Neurosci. 2013, 7, 267, doi:10.3389/fnins.2013.00267.

21. Boehler, C.; Stieglitz, T.; Asplund, M. Nanostructured platinum grass enables superior impedance reduction for neural microelectrodes. Biomaterials 2015, 67, 346-353, doi:10.1016/j.biomaterials.2015.07.036.

22. Górecka, J.; Makiewicz, P. The Dependence of Electrode Impedance on the Number of Performed EEG Examinations. Sensors (Basel) 2019, 19, doi:10.3390/s19112608.

23. Hostynek, J.J.; Maibach, H.I. Copper hypersensitivity: Dermatologic aspects. Dermatol. Ther. 2004, 17, 328-333, doi:10.1111/j.1396-0296.2004.04035.x.

24. Looney, D.; Kidmose, P.; Park, C.; Ungstrup, M.; Rank, M.; Rosenkranz, K.; Mandic, D. The in-the-ear recording concept: User-centered and wearable brain monitoring. IEEE Pulse 2012, 3, 32-42, doi:10.1109/MPUL.2012.2216717.

25. Grummett, T.S.; Leibbrandt, R.E.; Lewis, T.W.; DeLosAngeles, D.; Powers, D.M.W.; Willoughby, J.O.; Pope, K.J.; Fitzgibbon, S.P. Measurement of neural signals from inexpensive, wireless and dry EEG systems. Physiol. Meas. 2015, 36, 1469-1484, doi:10.1088/0967-3334/36/7/1469.

26. Christensen, C.B.; Harte, J.M.; Lunner, T.; Kidmose, P. Ear-EEG-Based Objective Hearing Threshold Estimation Evaluated on Normal Hearing Subjects. IEEE Trans. Biomed. Eng. 2018, 65, 1026-1034, doi:10.1109/TBME.2017.2737700. 